\title{
Risk factors for mortality in patients with Stenotrophomonas maltophilia bacteremia and clinical impact of quinolone-resistant strains
}

Eun Jin Kim¹, Yong Chan Kim¹, Jin Young Ahn², Su Jin Jeong ${ }^{2^{*}}$ (D, Nam Su Ku², Jun Yong Choi², Joon-Sup Yeom ${ }^{2}$ and Young Goo Song ${ }^{2}$

\begin{abstract}
Background: Stenotrophomonas maltophilia is an important nosocomial pathogen. This pathogen has intrinsic or acquired resistance to a number of antibiotics classes. Furthermore, Stenotrophomonas infections have been associated with high mortality, mainly in immunocompromised patients. Accordingly, we conducted a retrospective cohort study on the clinical data, microbiological characteristics, and outcomes of patients with S. maltophilia (SM) bacteremia.

Methods: A retrospective cohort study was conducted at two tertiary care referral hospitals in Seoul, South Korea. Data were collected between January 2006 and December 2015 from electric medical records. Our analysis aimed to identify the risk factors associated with crude mortality, as well as the predictive factors of quinolone-resistant strains in SM bacteremia patients.

Results: A total of 126 bacteremia patients were enrolled in the study. The mortality rate was $65.1 \%$. On multivariable analysis, hypoalbuminemia (odds ratio [OR], 5.090; 95\% confidence interval $[\mathrm{Cl}], 1.321-19.621 ; P=$ 0.018), hematologic malignancy $(\mathrm{OR}, 35.567 ; 95 \% \mathrm{Cl}, 2.517-502.515 ; P=0.008)$ and quinolone-resistant strains $(\mathrm{OR}, 7.785 ; 95 \% \mathrm{Cl}, 1.278-47.432 ; P=0.026)$ were independent risk factors for mortality. Alternatively, usage of an empirical regimen with quinolone $(\mathrm{OR}, 0.172 ; 95 \% \mathrm{Cl}, 0.034-0.875 ; P=0.034)$ was an independent protective factor for mortality. The multivariable analysis of predictive factors revealed that high Charlson comorbidity index (OR, 1.190; $95 \% \mathrm{Cl}, 1.040-1.361 ; P=0.011)$ and indwelling of a central venous catheter (CVC) (OR, 3.303; 95\% Cl, 1.194-9.139; $P=$ 0.021) were independent predisposing factors associated with quinolone-resistant strains in SM bacteremia patients.

Conclusions: Our findings suggest that a high Charlson comorbidity score and indwelling of a CVC were significantly independent predictors of quinolone-resistant strains in SM bacteremia patients. Therefore, we need to carefully consider the antibiotic use in SM bacteremia patients with these predictive factors.
\end{abstract}

Keywords: Quinolone-resistant strains, Stenotrophomonas maltophilia, Bacteremia

\footnotetext{
* Correspondence: JSJ@yuhs.ac

${ }^{2}$ Department of Internal Medicine and AIDS Research Institute, Yonse University College of Medicine, 50-1 Yonsei-ro, Seodaemun-gu, Seoul 120-752, Republic of Korea

Full list of author information is available at the end of the article
}

(c) The Author(s). 2019 Open Access This article is distributed under the terms of the Creative Commons Attribution 4.0 International License (http://creativecommons.org/licenses/by/4.0/), which permits unrestricted use, distribution, and reproduction in any medium, provided you give appropriate credit to the original author(s) and the source, provide a link to the Creative Commons license, and indicate if changes were made. The Creative Commons Public Domain Dedication waiver (http://creativecommons.org/publicdomain/zero/1.0/) applies to the data made available in this article, unless otherwise stated. 


\section{Background}

Stenotrophomonas maltophilia is a non-fermentative, gram-negative bacillus that is closely related to the Pseudomonas species. Bacterium bookeri, now known as $S$. maltophilia (SM), was first isolated in 1943 and was subsequently classified as a member of the genus Pseudomonas in 1961. Thereafter, it was classified as a member of Xanthomonas genus in 1983, and finally it came to rest in the Stenotrophomonas genus in 1993 [1]. SM is a bacterium that can occur in almost any aquatic or humid environment [2], and is not considered to be a highly virulent pathogen. Over the last decade, SM has risen to prominence as an important nosocomial pathogen associated with significant case/fatality ratios in certain patient populations, particularly in individuals who are severely debilitated or immunosuppressed [3-5]. Pneumonia and bacteremia are the most common manifestations of SM infection [6]. However, treatment of SM bacteremia is challenging due to the resistance of SM to many broad-spectrum antimicrobial agents. Moreover, SM exhibits high-level intrinsic resistance to a variety of structurally unrelated antibiotics, including: beta-lactams, quinolones, aminoglycosides, tetracycline, disinfectants, and heavy metals $[7,8]$. Furthermore, it can acquire resistance through the uptake of resistance genes located on integrons, transposons, and plasmids via horizontal gene transfer and mutations [9, 10]. Thus, choosing the optimal antibiotic for the treatment of SM bacteremia is very difficult. Trimethoprim-sulfamethoxazole (TMPSMX) should be considered as the empirical choice for clinically suspected SM infections and as the treatment of choice for culture-proven infections by this agent [11, 12]. However, due to concerns regarding adverse events related to TMP-SMX treatment, levofloxacin has also been used as an alternative option [13, 14]. Fluoroquinolone and SMX monotherapies may be equally effective for the treatment of SM infections [15]. But, the overuse of quinolones worldwide has resulted in higher resistance rates in SM [16-18]. Therefore, we investigated the predictive factors of quinolone-resistant strains in SM bacteremia patients.

\section{Methods}

\section{Study population and design}

A retrospective cohort study was conducted at two tertiary care referral hospitals in Seoul, South Korea. Data were collected between January 2006 and December 2014 from digital medical records. Patients 18 years or older with 1 or more positive blood cultures of SM that met the Centers for Disease Control and Prevention (CDC) criteria for blood stream infection (BSI) [19], were eligible for inclusion. If a patient had multiple episodes of bacteremia, only the data from the first episode were included.

\section{Definitions}

SM bacteremia was defined by the presence of a blood culture that yielded SM from one or more collected blood samples between January 2006 and December 2014. The source of bacteremia was determined clinically on the basis of the presence of an active site of infection as determined by chart review or isolation of the organism from other clinical specimens coincident with the episode of bacteremia. Polymicrobial bacteremia was defined by the isolation of an additional pathogen satisfying CDC criteria for BSI [19] within 24 h of index SM isolate. Healthcareassociated and community-acquired bacteremia were defined according to the CDC criteria for BSI [19]. Empiric antibiotic therapy was defined as the therapy initiated before the report of antibiotic susceptibility results, whereas definitive therapy was defined as the therapy given after the report of antibiotic susceptibility [20]. Chronic kidney disease was defined an estimated glomerular filtration rate (eGFR) (MDRD equation) of $<60 \mathrm{~mL} / \mathrm{min}$ per $1.73 \mathrm{~m}^{2}$ without renal replacement therapy. End stage renal disease was defined as an eGFR (MDRD equation) of $<15 \mathrm{~mL} /$ min per $1.73 \mathrm{~m}^{2}$ with renal replacement therapy. Pulmonary disease was defined as chronic obstructive lung disease or asthma. Appropriate antimicrobial therapy was defined as the administration of at least one agent to which the index SM isolate was susceptible in vitro. Immunosuppressive therapy was defined as a daily $\geq 20 \mathrm{mg}$ dose of a prednisolone-equivalent steroid, monoclonal antibodies, antimetabolite drugs, or T-cell inhibitors within 30 days prior to bacteremia onset. Neutropenia was defined as an absolute neutrophil count of $<500 / \mathrm{mm}^{3}$ at the onset of bacteremia. Thrombocytopenia was defined as a platelet count of less than $100,000 / \mathrm{mL}$, and hypoalbuminemia was defined as an albumin count of less than $3 \mathrm{~g} / \mathrm{dL}$ at the time of bacteremia. Mortality by SM bacteremia was defined as death within 60 days after the bacteremia isolation with no other apparent cause of death.

\section{Data collection}

A trained examiner used a electric medical record to collect demographic data. Data elements included demographics, length of hospital stay before SM bacteremia (days), source of bacteremia, antibiotics-susceptibility of the isolated pathogen, antimicrobial therapy regimen, past medical history, comorbid conditions, surgery, prior chemotherapy/radiation therapy, or receipt of immunosuppressive medications (each 30 days). The data included appropriateness of antibacterial therapy, existence of indwelling medical devices before SM bacteremia during hospitalization, removal of previously present CVC, and length of stay in ICU and hospital (days). The degree of comorbidity was quantified using the Charlson comorbidity index [21] and the severity of illness was assessed by the Acute Physiology and Chronic Health Evaluation II 
(APACHE II) score. Laboratory data and outcomes were also recorded.

\section{In vitro antimicrobial susceptibility testing}

All bacterial species were identified using conventional methods and/or the ATB 32GN system (bioMerieux, Marcy l'Etoile, France). Antimicrobial susceptibility tests were performed using the disk diffusion method or a VITEK-2 N131 card (bioMerieux, Hazelwood, MO, USA). The results were interpreted based on the Clinical and Laboratory Standards Institute (CLSI) guidelines [22]. SM isolates resistant to TMP-SMX were defined to have a minimal inhibitory concentration (MIC) $\geq 4 / 76 \mathrm{mg} / \mathrm{mL}$ to TMP-SMX. SM isolates which were resistant to levofloxacin (MIC $\geq 8 \mathrm{mg} / \mathrm{mL}$ ) or those with intermediate resistance (MIC $=4 \mathrm{mg} / \mathrm{mL}$ ) to levofloxacin were defined as quinolone-resistant strains.

\section{Statistical analysis}

All statistical analyses were performed using SPSS for Windows (ver. 23.0, SPSS Inc., Chicago, IL, USA). The statistical analyses were performed to assess the factors associated with crude mortality and quinolone susceptibility. Continuous variables were expressed as mean \pm standard deviation (SD), and categorical variables were expressed as a number (percentage). The KolmogorovSmirnov test was used to analyze the normality of the distribution of parameters. Data that did not show normal distributions were expressed as median and interquartile ranges (IQR). The Student's t-test was used for continuous variables and the Chi-square test or Fisher's exact test was used for categorical variables. Variables that did not show normal distributions were compared using the Mann-Whitney test or Kruskal-Wallis test. Univariate and multivariable logistic regression was used to evaluate the risk factors of mortality and the predictive factors of quinolone-resistant strains. Variables with $p$-values of less than 0.10 in univariate analyses were included in a multivariable logistic regression analysis to identify the risk factors associated with mortality and the predictive factors of quinolone-resistant strains. Results from the multivariate analysis are expressed as an odds ratio (OR) and 95\% confidence interval (CI). All statistical tests were two-tailed and a $P$ value of less than 0.05 was considered to be statistically significant.

\section{Results}

Baseline characteristics of SM bacteremia patients and comparison of clinical characteristics and outcomes

During the study period, 126 bacteremia patients were enrolled. The mean age of the patients was $61.22 \pm$ 15.07 years and the $68.3 \%(86 / 126)$ of the patients were men. The mean length of stay in the hospital before the occurrence of SM bacteremia was $57.1 \pm 22.5$ days. The mean Charlson comorbidity index score was $6.5 \pm 3.2$ and the median APACHE II score was 13.0 (IQR, 9-19).

Bivariable analyses were performed to investigate the risk factors of mortality in SM bacteremia patients. Table 1 shows a comparison of survivors and non-survivors during the entire hospitalization period. Univariate analyses, showed that hematologic malignancy $(P=$ $0.005)$, indwelling of hemodialysis catheter $(P=0.028)$, high APACHE II scores $(P=0.001)$, hypoalbuminemia $(P=0.003)$, thrombocytopenia $(P=0.004)$, and low hemoglobin concentration $(P=0.035)$ were associated with mortality. Also, the length of stay before bacteremia was longer in non-survivors $(P=0.038)$.

Anatomic origin and microbiologic findings of SM bacteremia are presented in Table 2. Catheter-related infection was the most common primary source of bacteremia (49 cases, 38.9\%), followed by intra-abdomen infection (38 case, 30.2\%) and respiratory infection (35 case, $27.8 \%$ ). The rate of CVC removal was 59.3\% (54/ 91). In our study, $11.9 \%(15 / 126)$ of patients had SM strains resistant to TMP/SMX, and 31.2\% (39/126) had strains resistant to quinolone. Only 21 patients (16.7\%) were using an empirical regimen with quinolone and there were no cases using an empirical regimen with TMP/SMX. The number of patients under a definitive regimen with quinolone was $32 \%$ (40/126), and with TMP/SMX it was $24.8 \%(31 / 126)$.

\section{Mortality risk factors in SM bacteremia patients}

On multivariable analysis, hypoalbuminemia (OR, 5.090; 95\% CI, 1.321-19.621; $P=0.018)$, hematologic malignancy (OR, 35.567; 95\% CI, 2.517-502.515; $P=0.008$ ) and quinolone-resistant strains (OR, 7.785; 95\% CI, 1.278-47.432; $P=0.026$ ) were independent risk factors for mortality. Contrary, usage of an empirical regimen with quinolone $(\mathrm{OR}, 0.172 ; 95 \% \mathrm{CI}, 0.034-0.875 ; P=$ $0.034)$ was independent protective factors for mortality (Table 3).

\section{Predicting factors for SM bacteremia with quinolone- resistant strains}

Analyses of predictive factors for SM bacteremia with quinolone-resistant strains were performed (Table 4). One patient died immediately on the day of bacteremia and was excluded from this analysis due to death not related to antibiotic adequacy ( $N=125$ cases). On univariate analysis, high Charlson comorbidity index and long lengths of hospital stay before the onset of bacteremia were significant related factors $(P=0.030$ and $P=0.015)$. Indwelling of CVC, ventilator, and Foley catheter were additional risk factors. Quinolone-resistant SM patients had a significantly higher mortality than the quinolone sensitive group ( $P=0.001$ and $P=0.013$ ). Based on this multivariable analysis, the Charlson comorbidity index (OR, 1.190; 95\% 
Table 1 Baseline characteristics of patients with S. maltophilia bacteremia

\begin{tabular}{|c|c|c|c|}
\hline Factors & Survivors $N=44$ & $\begin{array}{l}\text { Non-survivors } \\
N=82\end{array}$ & $p$ value \\
\hline Age, $y$, mean $\pm S D$ & $71.0(60.5-79)$ & $74.5(61.0-80.25)$ & 0.310 \\
\hline Age $\geq 65$ years, $n(\%)$ & $24(54.5)$ & $34(41.5)$ & 0.160 \\
\hline Male, n (\%) & $33(75.0)$ & $53(64.6)$ & 0.233 \\
\hline BMI, kg/m², median (IQR) & $21.7(19.4-25.0)$ & $21.9(19.0-24.4)$ & 0.847 \\
\hline \multicolumn{4}{|l|}{ Comorbidities } \\
\hline HTN, n (\%) & $15(34.1)$ & $32(39.0)$ & 0.585 \\
\hline $\mathrm{DM}, \mathrm{n}(\%)$ & $16(36.4)$ & $23(28.0)$ & 0.336 \\
\hline Cardiovascular disease, n (\%) & $9(20.5)$ & $13(15.9)$ & 0.517 \\
\hline Chronic kidney diseasea, n (\%) & $4(9.1)$ & $4(4.9)$ & 0.449 \\
\hline End stage renal disease, n (\%) & $1(2.3)$ & $7(8.5)$ & 0.259 \\
\hline Chronic liver disease, n (\%) & $5(11.4)$ & $11(13.4)$ & 0.742 \\
\hline Pulmonary disease, n (\%) & $4(9.1)$ & $8(9.8)$ & 1.000 \\
\hline Solid tumor, n (\%) & $18(40.9)$ & $43(52.4)$ & 0.217 \\
\hline Hematologic malignancy, n (\%) & $1(2.3)$ & $17(20.7)$ & 0.005 \\
\hline Solid organ transplantation, n (\%) & $3(6.8)$ & $4(4.9)$ & 0.694 \\
\hline Charlson score, mean \pm SD & $5.7 \pm 3.0$ & $6.9 \pm 3.2$ & 0.049 \\
\hline \multicolumn{4}{|l|}{ Predisposing factors } \\
\hline Chemotherapy, n (\%) & $4(9.1)$ & $10(12.2)$ & 0.769 \\
\hline Major surgerya ${ }^{a}, \mathrm{n}(\%)$ & $15(34.9)$ & $29(35.4)$ & 0.957 \\
\hline ICU care, n (\%) & $29(65.9)$ & $54(65.9)$ & 0.995 \\
\hline $\begin{array}{l}\text { Length of stay in hospital before } \\
\text { bacteremia (days), median (IQR) }\end{array}$ & $12.0(3.5-24.5)$ & $26.0(14.0-56.0)$ & 0.038 \\
\hline Central venous catheter, n (\%) & $31(70.5)$ & $59(72.8)$ & 0.777 \\
\hline Hemodialysis catheter, n (\%) & $2(4.5)$ & $15(18.8)$ & 0.028 \\
\hline Mechanical ventilator, n (\%) & $19(43.2)$ & $41(50.6)$ & 0.427 \\
\hline Foley catheter, n (\%) & $28(63.6)$ & $47(58.0)$ & 0.541 \\
\hline \multicolumn{4}{|l|}{ Clinical severity } \\
\hline Shock, n (\%) & $10(22.7)$ & $33(40.2)$ & 0.075 \\
\hline APACHE II score, mean \pm SD & $11.0 \pm 5.8$ & $15.4 \pm 6.9$ & $<0.001$ \\
\hline \multicolumn{4}{|l|}{ Laboratory findings } \\
\hline Neutropenia, n (\%) & $3(6.8)$ & $15(18.3)$ & 0.079 \\
\hline Hypoalbuminemia, n (\%) & $18(6.8)$ & $56(18.3)$ & 0.003 \\
\hline Thrombocytopenia, n (\%) & $9(20.5)$ & $38(46.3)$ & 0.004 \\
\hline Hemoglobin $(\mathrm{g} / \mathrm{L})$, mean $\pm \mathrm{SD}$ & $10.1 \pm 1.6$ & $9.4 \pm 1.5$ & 0.032 \\
\hline C-reactive protein (mg/L), median (IQR) & $69.2(32.2-129.5)$ & $89.3(58.5-148.0)$ & 0.057 \\
\hline $\begin{array}{l}\left.\text { Estimated GFR (ml/min per } 1.73 \mathrm{~m}^{2}\right) \text {, } \\
\text { median (IQR) }\end{array}$ & $85.0(54.2-98.0)$ & $80.0(44.0-104.0)$ & 0.675 \\
\hline Hospital stay, days & $53.0(20.5-78.0)$ & $60.0(35.0-97.0)$ & 0.137 \\
\hline Length of stay in ICU, days & $30(11.5-50.0)$ & $39(23.0-67.0)$ & 0.640 \\
\hline
\end{tabular}

Note. SD Standard deviation, BMI Body mass index, IQR Interquartile range, HTN Hypertension, DM Diabetes mellitus, ICU Intensive care unit, APACHE II Acute Physiologic and Chronic Health Evaluation II score

${ }^{a}$ Major surgery, any surgical procedure that involves anesthesia or respiratory assistance 
Table 2 Anatomic origin and microbiologic findings of S. maltophilia bacteremia in study participants

\begin{tabular}{|c|c|c|c|}
\hline Factors & Survivors $N=44$ & Non-survivors $N=82$ & $p$ value \\
\hline \multicolumn{4}{|l|}{ Infection source } \\
\hline Pneumonia & $12(27.9)$ & $23(28.0)$ & 0.987 \\
\hline Catheter-related infection & $15(34.1)$ & $34(42.0)$ & 0.202 \\
\hline Intra-abdominal infection & $16(36.4)$ & $22(27.2)$ & 0.285 \\
\hline Soft tissue infection & $0(0.0)$ & $5(6.2)$ & 0.161 \\
\hline Urinary tract infection & $1(2.3)$ & $0(0.0)$ & 0.352 \\
\hline Polymicrobial infection & $18(40.9)$ & $36(43.9)$ & 0.746 \\
\hline \multicolumn{4}{|l|}{ Antibiotics susceptibility } \\
\hline Quinolone resistance & $5(11.6)$ & $34(41.5)$ & 0.001 \\
\hline TMP-SMX resistance & $3(6.8)$ & $12(14.6)$ & 0.197 \\
\hline Resistant strain ${ }^{a}$ & 17 (38.6) & 49 (59.8) & 0.024 \\
\hline \multicolumn{4}{|l|}{ Treatment } \\
\hline \multicolumn{4}{|l|}{ Empirical antibiotic use } \\
\hline Cephalosporins & $2(4.5)$ & $6(7.4)$ & 0.711 \\
\hline Carbapenems & $13(29.5)$ & $42(51.9)$ & 0.016 \\
\hline Fluoroquinolones & $13(29.5)$ & $8(9.8)$ & 0.004 \\
\hline BLBLIS & $14(31.8)$ & $16(19.5)$ & 0.122 \\
\hline TMP-SMX & $0(0.0)$ & $0(0.0)$ & - \\
\hline \multicolumn{4}{|l|}{ Definitive antibiotic use } \\
\hline Carbapenems & $2(4.5)$ & $16(19.5)$ & 0.022 \\
\hline Fluoroquinolones & $21(47.7)$ & $19(23.5)$ & 0.005 \\
\hline BLBLIS & $3(6.8)$ & $5(6.2)$ & 1.000 \\
\hline TMP-SMX & $4(9.1)$ & $27(33.3)$ & 0.003 \\
\hline Inappropriate antimicrobial therapy ${ }^{\mathrm{b}}$, yes & $14(31.8)$ & $25(30.9)$ & 0.912 \\
\hline
\end{tabular}

Note. BLBLIs Beta-lactam/beta-lactamase inhibitors, TMP-SMX Trimethoprim-sulfamethoxazole

The data were expressed as number (\%) or median (interquartile range)

${ }^{a}$ Resistant strain, Quinolone or/and TMP-SMX resistance

${ }^{\mathrm{b}}$ Appropriate antimicrobial therapy, the administration of at least one agent to which the index SM isolate was susceptible in vitro

CI, 1.040-1.361; $P=0.011)$ and indwelling of a CVC (OR, 3.303; 95\% CI, 1.194-9.139; $P=0.021$ ) were identified as independent predisposing factors associated with quinoloneresistant strains in SM bacteremia patients.

\section{Discussion}

Among non-fermenting gram negative bacilli, SM has been reported to be the third most commonly isolated pathogen after Pseudomonas aeruginosa and Acinetobacter baumannii [23]. Furthermore, SM bacteremia is associated with

Table 3 Multivariate analysis for predictive factors for mortality in patients with S. maltophilia bacteremia

\begin{tabular}{llll}
\hline Factors & OR & $95 \% \mathrm{Cl}$ & $P$ value \\
\hline Hematologic malignancy & 35.57 & $2.52-502.52$ & 0.008 \\
Hypoalbuminemia & 5.09 & $1.32-19.62$ & 0.018 \\
Quinolone resistance & 7.79 & $1.28-47.43$ & 0.026 \\
Empirical antibiotic - quinolone use & 0.17 & $0.03-0.88$ & 0.034
\end{tabular}

Note. OR Odds ratio, $\mathrm{Cl}$ Confidence interval high mortality rates, the crude mortality estimates range from 21 to $69 \%$ [23]. Previous studies have reported that risk factors associated with mortality for SM bacteremia include indwelling of CVC in intensive care units, immunocompromising conditions, exposure to broad-spectrum antibiotic therapy, and long hospital stays $[3,9,23]$. In our study, the mortality rate of $65.1 \%$ was similar to the rate reported in previous studies. Bivariable analyses to investigate the risk factors of mortality in SM bacteremia, identified hematologic malignancy and hypoalbuminemia as independent risk factors. In addition, our results indicate that quinolone resistance has an impact on mortality, and it has also been shown that the use of empirical quinolone can reduce mortality after adjusting the analysis. There were also statistical differences in crude $(87.2 \%$ vs $55.8 \%, P=0.001)$ and attributable mortality $(61.5 \%$ vs $30.6 \%, P=0.001)$ between quinolone-resistant and sensitive groups. Consequently, we analyzed the risk factors associated with quinolone resistance in SM bacteremia. To our knowledge, there are no reports investigating the risk factors for 
Table 4 Comparisons of Clinical characteristics between quinolone-susceptible and quinolone-resistant groups

\begin{tabular}{|c|c|c|c|}
\hline Factors & Susceptible group $N=86$ & Resistant group $N=39$ & $p$ value \\
\hline Age, years & $63.0(51.0-70.0)$ & $66.0(53.5-72.5)$ & 0.215 \\
\hline Age $\geq 65$ years, $n(\%)$ & $37(43.0)$ & $20(51.3)$ & 0.390 \\
\hline Gender, male & $57(66.3)$ & $29(64.6)$ & 0.233 \\
\hline \multicolumn{4}{|l|}{ Comorbidities } \\
\hline HTN & $30(34.9)$ & $16(41.0)$ & 0.509 \\
\hline DM & $29(33.7)$ & $10(25.6)$ & 0.366 \\
\hline Cardiovascular disease & $13(15.1)$ & $9(23.1)$ & 0.279 \\
\hline Chronic kidney disease & $6(7.0)$ & $2(5.1)$ & 1.000 \\
\hline End stage renal disease & $4(4.7)$ & $4(10.3)$ & 0.255 \\
\hline Chronic liver disease & $12(14.0)$ & $4(10.3)$ & 0.774 \\
\hline Pulmonary disease & $8(9.3)$ & $4(10.3)$ & 1.000 \\
\hline Solid tumor & $42(48.8)$ & $19(48.7)$ & 0.990 \\
\hline Hematologic malignancy & $12(14.0)$ & $6(15.4)$ & 0.833 \\
\hline Solid organ transplantation & $5(5.8)$ & $2(5.1)$ & 1.000 \\
\hline Charlson score & $6.1 \pm 3.1$ & $7.4 \pm 3.2$ & 0.030 \\
\hline \multicolumn{4}{|l|}{ Predisposing factors } \\
\hline Chemotherapy & $11(12.8)$ & $3(7.7)$ & 0.546 \\
\hline Major surgery ${ }^{a}$ & $28(32.9)$ & $16(35.4)$ & 0.382 \\
\hline ICU care & $54(62.8)$ & $29(74.4)$ & 0.205 \\
\hline Hospital stay before bacteremia, days & $17.0(8.0-34.0)$ & $30.0(13.5-57.5)$ & 0.015 \\
\hline Central venous catheter & $57(66.3)$ & $32(84.2)$ & 0.019 \\
\hline Hemodialysis catheter & $9(10.5)$ & $8(21.6)$ & 0.100 \\
\hline Mechanical ventilator & $36(41.9)$ & $24(63.2)$ & 0.029 \\
\hline Foley catheter & $47(54.7)$ & $28(73.7)$ & 0.046 \\
\hline \multicolumn{4}{|l|}{ Clinical findings } \\
\hline Shock & $23(26.7)$ & $20(51.3)$ & 0.007 \\
\hline APACHE II score & $13.0(8.0 ; 18.0)$ & $14.0(11.0 ; 21.5)$ & 0.115 \\
\hline \multicolumn{4}{|l|}{ Laboratory findings } \\
\hline Neutropenia & $14(16.3)$ & $4(10.3)$ & 0.374 \\
\hline Hypoalbuminemia & $52(60.5)$ & $21(53.8)$ & 0.487 \\
\hline Thrombocytopenia & $28(32.6)$ & $19(48.7)$ & 0.084 \\
\hline Hemoglobin, $g / L$ & $9.8 \pm 1.7$ & $9.4 \pm 1.3$ & 0.296 \\
\hline C-reactive protein, mg/L & $86.8(41.5-142.5)$ & $79.3(44.5-153.0)$ & 0.914 \\
\hline Estimated GFR, $\mathrm{ml} / \mathrm{min}$ per $1.73 \mathrm{~m}^{2}$ & $87.0(51.0-101.0)$ & $72.0(45.4-106.0)$ & 0.592 \\
\hline \multicolumn{4}{|l|}{ Outcomes } \\
\hline Inappropriate antimicrobial therapy & $23(26.7)$ & $16(42.1)$ & 0.089 \\
\hline Hospital stay, days & $50.0(26.0-78.0)$ & $68.0(35.0-150.0)$ & 0.054 \\
\hline Mortality & $48(55.8)$ & 34 (87.2) & 0.001 \\
\hline
\end{tabular}

Note. HTN Hypertension, DM Diabetes mellitus, ICU Intensive care unit, APACHE II Acute Physiologic and Chronic Health Evaluation II score

The data were expressed as mean \pm standard deviation, number (\%), or median (interquartile range)

${ }^{a}$ Major surgery, any surgical procedure that involves anesthesia or respiratory assistance

quinolone-resistant SM bacteremia. There have been studies of risk factors related to the acquisition of resistance to levofloxacin in all clinical specimens and to quinolone susceptibility using only respiratory tract specimens with SM
$[17,24]$. In respiratory tract specimens, a relative factor for resistance to quinolone was the previous use of piperacillin/ tazobactam, and risk factors to levofloxacin resistance were exposure to levofloxacin for more than 3 weeks and co- 
infection/co-colonization with Klebsiella pneumoniae resistant to levofloxacin.

In this report, a high Charlson comorbidity index and indwelling of CVC were independent risk factors associated with resistance to quinolone in SM bacteremia. The Charlson comorbidity index is a widely used comorbidity index [21]. A high CCI value means that many coexistent diseases may directly or indirectly affect the choice of antibiotics used and the outcome [25]. Especially, the presence of many coexistent diseases suggests that the previous use of antibiotics, or a prolonged history of hospital admissions, provides opportunities for coexistent diseases to develop. Furthermore, a high CCI value is associated with a greater risk to acquire common antibiotic resistance. This may be due to previous antibiotic uptake or hospital stays, however, this analysis was not part of our study and further studies are needed.

Indwelling of CVC has already been shown to be significantly associated with mortality and bacteremia in previous literature [9]. In particular, removal of CVC has been associated with reduced mortality [26-28]. However, removal of CVC did not influence mortality and quinolone resistance in this report. It is estimated that the rate of patients who adequately removed CVC was only $40 \%$, and therefore did not significantly affect the outcome. SM has the ability to adhere on prosthetic devices such as CVC and form a biofilm. Biofilms can increase antibiotic resistance [11]. Therefore, indwelling of $\mathrm{CVC}$ is a risk factor to biofilm formation and, accordingly, to quinolone-resistance. Therefore, it is necessary to actively encourage the removal of CVC.

Previous literature has proven the relationship between inappropriate antimicrobial therapy and mortality in SM infections [23, 27]. Although not relevant to this study, usage of empirical quinolone had a significantly reduced mortality risk. Empirical antibiotic regimens are determined by the severity of patients. Therefore, patients that underwent an empirical quinolone regimen were more likely to have low SM severity and lower mortality. Overall, the periods of empirical antibiotic use were short and there were no patients using TMP / SMX empirically, so whether the empirical use of quinolone actually had a significant impact needs to be analyzed further. Also, SM infections may not be an independent contributor to mortality increase, therefore inappropriate therapy should not have major effects on the outcome of patients [29]. The rate of polymicrobial infections in SM bacteremia was high in the previous literature [23] and in this study as well (42.8\%). Thus, high polymicrobial infections may be a confounding factor for appropriate antibiotic use, and this may have affected the mortality risk-factor analysis. Therefore, subgroup analysis was performed in patients with polymicrobial infection. However, there was no significant difference between the two groups regarding mortality (Additional file 1: Table S1).
Significantly, the use of empirical or definitive treatment of carbapenem was higher in the mortality group, although it was not significant in the multivariate analysis. This suggests that the possibility of breakthrough infections by SM in patients being treated with carbapenem is due to intrinsically resistant carbapenem and the selection pressure of SM should be considered [29, $30]$. Only $24.8 \%$ of patients received TMP/SMX. A high proportion of ICU care and polymicrobial infections may have influenced antibiotic selection. Nevertheless, careful use of carbapenem is necessary, and the possibility for breakthrough infections should be considered.

Our study had several limitations. First, the retrospective design of this study was a major limitation. Second, the history of previous antibiotic use and previous hospital admissions were not investigated. This is especially important, since these are factors related to the acquisition of antibiotic resistance. Third, we could not investigate the susceptibility of other antibiotics except quinolone and TMP/SMX, and our quinolone analysis was limited to levofloxacin only.

\section{Conclusion}

Quinolone-resistant SM isolates have been emerging and spreading in Korean hospitals, and current therapeutic options are limited for SM bacteremia. Our results suggest that a high Charlson comorbidity index and indwelling of CVC were significant independent predictors of SM bacteremia patients with quinolone-resistant strains. Therefore, we need to carefully consider antibiotics use in patients with SM bacteremia who have these predictive factors.

\section{Additional file}

Additional file 1: Table S1. Clinical characteristics of polymicrobial infection groups. (DOCX 22 kb)

\section{Abbreviations \\ APACHE II: Acute Physiology and Chronic Health Evaluation II; BSI: Blood stream infection; CDC: Centers for Disease Control and Prevention; Cl: Confidence interval; CVC: Central venous catheter; eGFR: Estimated glomerular filtration rate; IQR: Interquartile range; MIC: Minimal inhibitory concentration; OR: Odds ratio; SD: Standard deviation; SM: S. maltophilia; TMP-SMX: Trimethoprim-sulfamethoxazole}

\section{Acknowledgements}

This abstract has previously been presented as part of a poster below. Eun Jin Kim, et al. "Predictive Factors Associated With Stenotrophomonas maltophilia Bacteremia Infected by Antibiotic-Resistant Strains." Open Forum Infectious Diseases. Vol. 3. No. suppl_1. Oxford University Press, 2016.

\section{Authors' contributions}

Conception and design of study: EJK, SJJ Acquisition of data: EJK, YCK, JYA NSK Data analysis and interpretation: EJK, SJJ Drafting of manuscript and critical revision: EJK, SJJ, JYC, JSY, YGS Approval of final version of manuscript: EJK, YCK, JYA, SJJ, NSK, JYC, JSY, YGS. 


\section{Funding}

This Research did not receive any specific grant from funding agencies in the public, commercial, or not-for-profit sectors.

\section{Availability of data and materials}

The datasets used during the current study are available from the corresponding author on reasonable request.

\section{Ethics approval and consent to participate}

The study was approved by the institutional review board of the Yonsei University Health System Clinical Trial Center. The informed consent was waived because this study was a retrospective study with review of related data through the electronic medical record. The datasets used and/or analyzed during the current study available from the corresponding author on reasonable request.

\section{Consent for publication}

Not applicable.

\section{Competing interests}

The authors declare that they have no competing interests.

\section{Author details}

Department of infectious diseases, Ajou University School of Medicine, Suwon, Korea. ${ }^{2}$ Department of Internal Medicine and AIDS Research Institute, Yonsei University College of Medicine, 50-1 Yonsei-ro, Seodaemun-gu, Seoul 120-752, Republic of Korea.

Received: 26 March 2019 Accepted: 20 August 2019

Published online: 28 August 2019

\section{References}

1. Denton M, Kerr KG. Microbiological and clinical aspects of infection associated with Stenotrophomonas maltophilia. Clin Microbiol Rev. 1998; 11(1):57-80

2. Hoefel D, Monis PT, Grooby WL, Andrews S, Saint CP. Profiling bacterial survival through a water treatment process and subsequent distribution system. J Appl Microbiol. 2005;99(1):175-86.

3. Falagas ME, Kastoris AC, Vouloumanou EK, Rafailidis PI, Kapaskelis AM, Dimopoulos G. Attributable mortality of Stenotrophomonas maltophilia infections: a systematic review of the literature. Future Microbiol. 2009;4(9): 1103-9.

4. Micozzi A, Venditti M, Monaco M, Friedrich A, Taglietti F, Santilli S, et al. Bacteremia due to Stenotrophomonas maltophilia in patients with hematologic malignancies. Clin Infect Dis. 2000;31(3):705-11.

5. Labarca JA, Leber AL, Kern VL, Territo MC, Brankovic LE, Bruckner DA, et al. Outbreak of Stenotrophomonas maltophilia bacteremia in allogenic bone marrow transplant patients: role of severe neutropenia and mucositis. Clin Infect Dis. 2000;30(1):195-7.

6. Gales AC, Jones RN, Forward KR, Linares J, Sader HS, Verhoef J. Emerging importance of multidrug-resistant Acinetobacter species and Stenotrophomonas maltophilia as pathogens in seriously ill patients: geographic patterns, epidemiological features, and trends in the SENTRY antimicrobial surveillance program (1997-1999). Clin Infect Dis. 2001; 32(Suppl 2):S104-13.

7. Zhang L, Li XZ, Poole K. Multiple antibiotic resistance in Stenotrophomonas maltophilia: involvement of a multidrug efflux system. Antimicrob Agents Chemother. 2000:44(2):287-93.

8. Alonso A, Martinez JL. Multiple antibiotic resistance in Stenotrophomonas maltophilia. Antimicrob Agents Chemother. 1997;41(5):1140-2.

9. Looney WJ, Narita M, Muhlemann K. Stenotrophomonas maltophilia: an emerging opportunist human pathogen. Lancet Infect Dis. 2009;9(5):312-23.

10. Sanchez MB. Antibiotic resistance in the opportunistic pathogen Stenotrophomonas maltophilia. Front Microbiol. 2015;6:658.

11. Nicodemo AC, Paez Jl. Antimicrobial therapy for Stenotrophomonas maltophilia infections. Eur J Clin Microbiol Infect Dis. 2007;26(4):229-37.

12. Chung HS, Hong SG, Kim YR, Shin KS, Whang DH, Ahn JY, et al. Antimicrobial susceptibility of stenotrophomonas maltophilia isolates from Korea, and the activity of antimicrobial combinations against the isolates. $J$ Korean Med Sci. 2013;28(1):62-6.
13. Cho SY, Kang Cl, Kim J, Ha YE, Chung DR, Lee NY, et al. Can levofloxacin be a useful alternative to trimethoprim-sulfamethoxazole for treating Stenotrophomonas maltophilia bacteremia? Antimicrob Agents Chemother. 2014:58(1):581-3.

14. Bonfiglio G, Cascone C, Azzarelli C, Cafiso V, Marchetti F, Stefani S. Levofloxacin in vitro activity and time-kill evaluation of Stenotrophomonas maltophilia clinical isolates. J Antimicrob Chemother. 2000;45(1):115-7.

15. Wang YL, Scipione MR, Dubrovskaya Y, Papadopoulos J. Monotherapy with fluoroquinolone or trimethoprim-sulfamethoxazole for treatment of Stenotrophomonas maltophilia infections. Antimicrob Agents Chemother. 2014:58(1):176-82.

16. Chang YT, Lin CY, Lu PL, Lai CC, Chen TC, Chen CY, et al. Stenotrophomonas maltophilia bloodstream infection: comparison between community-onset and hospital-acquired infections. Wei mian yu gan ran za zhi. 2014;47(1):28-35.

17. Pien CJ, Kuo HY, Chang SW, Chen PR, Yeh HW, Liu CC, et al. Risk factors for levofloxacin resistance in Stenotrophomonas maltophilia from respiratory tract in a regional hospital. Wei mian yu gan ran za zhi. 2015;48(3):291-5.

18. Wu H, Wang JT, Shiau YR, Wang HY, Lauderdale TL, Chang SC. A multicenter surveillance of antimicrobial resistance on Stenotrophomonas maltophilia in Taiwan. Wei mian yu gan ran za zhi. 2012;45(2):120-6.

19. Horan TC, Andrus M, Dudeck MA. CDC/NHSN surveillance definition of health care-associated infection and criteria for specific types of infections in the acute care setting. Am J Infect Control. 2008;36(5):309-32.

20. McGregor JC, Rich SE, Harris AD, Perencevich EN, Osih R, Lodise TP Jr, et al. A systematic review of the methods used to assess the association between appropriate antibiotic therapy and mortality in bacteremic patients. Clin Infect Dis. 2007:45(3):329-37.

21. Charlson ME, Pompei $P$, Ales KL, Mackenzie CR. A new method of classifying prognostic comorbidity in longitudinal studies: development and validation. J Chronic Dis. 1987:40(5):373-83.

22. Patel JB, Cockerill FR III, Bradford PA, Eliopoulos GM, Hindler JA, Jenkins SG, et al. Clinical and laboratory standards institute. Performance standards for antimicrobial susceptibility testing; 25 th informational supplementvol. 35 Number 3: CLSI; 2015: (Document M100-S124) CLSI; 2015.

23. Paez Jl, Costa SF. Risk factors associated with mortality of infections caused by Stenotrophomonas maltophilia: a systematic review. J Hosp Infect. 2008; 70(2):101-8.

24. Baek JH, Kim CO, Jeong SJ, Ku NS, Han SH, Choi JY, et al. Clinical factors associated with acquisition of resistance to levofloxacin in Stenotrophomonas maltophilia. Yonsei Med J. 2014;55(4):987-93.

25. Ma HY, Hung IC, Huang YH, Chang YY, Sheng WH, Wang JT, et al. Prognostic factors of health care-associated bloodstream infection in adult patients $>/=40$ years of age. Am J Infect Control. 2018:46(1):111-4.

26. Araoka H, Baba M, Yoneyama A. Risk factors for mortality among patients with Stenotrophomonas maltophilia bacteremia in Tokyo, Japan, 1996-2009. Eur J Clin Microbiol Infect Dis. 2010;29(5):605-8.

27. Friedman ND, Korman TM, Fairley CK, Franklin JC, Spelman DW. Bacteraemia due to Stenotrophomonas maltophilia: an analysis of 45 episodes. J Infect. 2002:45(1):47-53.

28. Jeon YD, Jeong WY, Kim MH, Jung IY, Ahn MY, Ann HW, et al. Risk factors for mortality in patients with Stenotrophomonas maltophilia bacteremia. Medicine. 2016;95(31):e4375

29. Ebara H, Hagiya H, Haruki Y, Kondo E, Otsuka F. Clinical characteristics of Stenotrophomonas maltophilia bacteremia: a regional report and a review of a Japanese case series. Internal Med. 2017;56(2):137-42.

30. Sanyal SC, Mokaddas EM. The increase in carbapenem use and emergence of Stenotrophomonas maltophilia as an important nosocomial pathogen. J Chemother. 1999:11(1):28-33.

\section{Publisher's Note}

Springer Nature remains neutral with regard to jurisdictional claims in published maps and institutional affiliations. 